\title{
A Classification System Specific for Recurrent Inguinal Hernia Following Open Hernia Surgery
}

\author{
NAOTAKA YAMAGUCHI ${ }^{1,2}$, DAISUKE MORIOKA ${ }^{1,2}$, YUSUKE IZUMISAWA $^{1}$, \\ JUNYA TOYODA $^{1}$, KAZUYA YAMAGUCHI ${ }^{1}$, NOBUTOSHI HORII ${ }^{1}$, NORIO OHYAMA ${ }^{1}$, FUMIO ASANO ${ }^{1}$, \\ MASARU MIURA ${ }^{1}$, RYUSEI MATSUYAMA $^{1,2}$, YOSHIKI SATO $^{1}$ and ITARU ENDO ${ }^{2}$ \\ ${ }^{I}$ Department of Surgery, Yokohama Ekisaikai Hospital, Yokohama, Japan; \\ ${ }^{2}$ Department of Gastroenterological Surgery, \\ Yokohama City University Graduate School of Medicine, Yokohama, Japan
}

\begin{abstract}
Background/ Aim: Currently, there is no classification system specializing in recurrent inguinal hernia (RIH) after open-surgery. For this reason, in this study we proposed one so as to understand the causes of RIH. Patients and Methods: Recurrence of IH after suturerepair was classified either as the tissue-loosening (TL) or the tissue-disruption (TD) type. Recurrence after open-meshrepair was classified according to the locational relation between the hernia-defect and the mesh, as follows: i) meshdistant (MD), ii) para-mesh (PM), iii) mesh-migration (MM), and iv) unclassifiable (UC). Fifty-two RIHs in 48 patients were classified, using this system, and analyzed. Results: This system-based classification led to the identification of: i) $M M$ in 11 lesions, ii) PM in 11, iii) $M D$ in 10, iv) TL in 7, v) TD in 5, and vi) UC in 8 lesions. The median time to recurrence (MTR) was significantly shorter in patients who had previously undergone a mesh-repair $(n=34)$ compared to those who had undergone a suture-repair $(n=13)$ [Meshrepair vs. suture-repair MTR: 1.6 years (0.1-20) vs. 30 years (15-72), $p<0.001]$. MTR was significantly shorter in the following order: i) $M M$ [0.5(0.1-2.0)]), ii) PM [2.6(0.2-15)]), iii) $M D[11(0.5-20)]$, iv) TD [20(15-30)], and v) TL [40(3072)] ( $p<0.001)$. Conclusion: This classification system helps understand the causes of RIH, leading to improved outcomes following open-surgery in the future.
\end{abstract}

This article is freely accessible online.

Correspondence to: Daisuke Morioka, Department of Surgery, Yokohama Ekisaikai Hospital 1-2 Yamada-cho, Naka-ku, Yokohama 231-0036, Japan. Tel: +81 452618191, Fax: +81 452618149, e-mail: dmorioka@hotmail.com

Key Words: Cause of recurrence, classification system, recurrent inguinal hernia.
Open inguinal hernia repair (IHR) continues to be a common procedure as it is more cost-effective than the laparoscopic IHR (1-3). Although acute/chronic postoperative pain has been reportedly more common in the open IHR than in the laparoscopic IHR, the incidence of recurrent inguinal hernia (RIH) has been, reportedly, similar following either of these two procedures $(2,4-6)$, and open IHR still accounts for the bulk of training procedures $(2,7)$. A reported lifetime risk of inguinal hernia $(\mathrm{IH})$ can be regarded as markedly high, at approximately $30 \%$ for men and $5 \%$ for women worldwide $(2,8,9)$.

The RIH is associated with the interplay between i) nonmodifiable, patient-related, and ii) modifiable, prior-tooperation-related factors $(2,10-12)$. The increased vulnerability of the abdominal wall due to aging is considered as a representative factor among the nonmodifiable ones. Meanwhile, overlooking some types of multiple hernia defects, e.g., combined indirect and direct hernias, is considered as a representative of the modifiable factors (13). In addition, inappropriate mesh handling, such as distortion, migration, and/or shifted placement of the mesh, is considered as another representative of the modifiable factors (13).

Unlike life-threatening conditions, the underlying causes of RIH have not been thoroughly analyzed $(2,10-12)$. The most prevalent classification system for IH, the European Hernia Society classification (EHSC), notates IHs irrespective of primary $(\mathrm{P})$ or recurrent $(\mathrm{R})$ hernias except for the notation 'P-' or 'R-' (1), meaning that the mechanisms of recurrence are not reflected into the EHSC at all. Moreover, it seems that most surgeons do not have a clear understanding of how RIH develops when they encounter it, probably because a detailed classification system specializing in RIH does not exist.

Herein, we propose a classification system specific for RIH after open IHR and analyze its clinical relevance to verify whether: i) the RIH after mesh-repair develops earlier 
than the recurrence after suture-repair, ii) the RIH caused by modifiable factors develops earlier than the recurrence caused by non-modifiable factors, and iii) the more inappropriate the mesh handling is, the earlier the recurrence is. We believe that this system reflects the mechanisms of developing $\mathrm{RIH}$, thus leading to improved outcomes in future primary open IHR.

\section{Patients and Methods}

This study was approved by the Institutional Review Board (IRB) (Approval No. YEH2014-S-01), and was compliant with the Declaration of Helsinki.

Patients. From April 2014 to March 2019, we performed surgery for 52 RIH lesions in 48 patients over the age of 18 years, who had previously undergone open IHR. These 52 cases were included in this study. Pediatric IHR cases under the age of 18 years were excluded. The following variables were prospectively collected and retrospectively analyzed: i) age at the time of surgery for recurrence, ii) gender, iii) age at primary IHR, iv) time to recurrence, v) EHSC for the primary and RIH, vi) methods of the first IHRs, vii) methods of the RIH surgery, viii) use of a laparoscope during RIH surgery, ix) the RIH classification according to the EHSC and the $x$ ) classification system described below, as well as xi) the clinical course following RIH surgery.

The classification system. Recurrence after the non-mesh suturerepair or Lichtenstein-patch repair (LPR) was classified as: i) the tissue-loosening type (TL) (Figure 1A) and ii) the tissue-disruption type (TD) (Figure 1B), depending on whether the continuity of the posterior floor tissue was maintained or not. The TL is considered to be caused by abdominal wall loosening due to the alterations in patients' bodies, while the TD is literally attributed to a disruption in the posterior floor of the inguinal canal.

Recurrence after the primary open-posterior-mesh-repair (OPMR) was classified according to the locational relation between the hernia defect(s) and a previously indwelled mesh, such as : i) the mesh-distant type (MD) (Figure 1C), in which the hernia defect is distant from the indwelled mesh; ii) the para-mesh type (PM) (Figure 1D), in which the hernia defect is adjacent to the indwelled mesh; iii) the mesh-migration type (MM) (Figure 1E), in which the mesh has migrated from the place where it should have been placed and/or the mesh is obviously distorted and found away from the abdominal wall.

In either the PM or the MD type, the indwelled mesh should not be distorted. The causes of the MD type are speculated to be due to: i) the laxness of the abdominal wall distant from the indwelled mesh developing after the primary surgery and ii) to overlooking certain cases of multiple hernia defects. The causes of the PM type can be considered to be due to: i) the progressively increased vulnerability of the abdominal wall surrounding the indwelled mesh and ii) an inappropriate mesh usage, such as a too-small mesh and/or a shifted mesh placement. The MM type can be regarded as the representative of an inappropriate surgical procedure because it cannot develop unless the previously indwelled mesh has not been placed properly.

Recurrence that could not be classified as one of the five abovedescribed types was defined as the unclassifiable type (UC).
A time-phase of the RIH was defined according to the duration from the primary open IHR to the recurrence as follows: i) early (E, $\leq 2$ years), ii) intermediate (I, $2<$ and $\leq 20$ years), and iii) late ( $\mathrm{L}$, $>20$ years). The time for developing RIH was determined depending on the time when patients realized their recurrent inguinal bulging following the first surgery.

The notation of this system was determined as (type)-(timephase): e.g., TL-L, MM-E, etc. If multiple hernia defects were identified, the notation of each defect was concurrently noted as (type)-(type)-(time-phase): e.g., MD-PM-I, MM-MD-E, etc.

We initiated laparoscopic IHR in January 2015 when our IRB approved this procedure. Thus, the hybrid method, including the mesh placement in the preperitoneal space through the anterior approach with the aid of laparoscopic view (14), has been performed since then. Exclusive laparoscopic RIH repair (15) has been performed since April 2016, when our IRB approved the procedure. Cases of exclusive laparoscopic repair for RIH, whether the previous IHR was LPR or not, were confirmed by laparoscopic inspection and/or palpation using laparoscopic-surgical forceps through hernia defect(s), following exfoliation of the parietal peritoneum.

The correlation between this system-based classification and other clinical variables were analyzed.

Statistical analyses. Summary statistics are shown as median [range or interquartile range (IQR)] or numbers and/or percentages. Continuous variables were compared using the Mann-Whitney $U$-test or Kruskal-Wallis test and categorical variables were compared using Fisher's exact probability test. The cumulative re-recurrence rate was estimated using the Kaplan-Meier method and compared using the Breslow-Gehan-Wilcoxon test in March 31, 2020. The data were analyzed using the software program SPSS ver. 23 (Armonk, NY, USA). Two-tailed $p$-Values of $<0.05$ were accepted as significant.

\section{Results}

Patient characteristics. Patient characteristics are summarized in Table I. The median time to recurrence (MTR) from the primary IHR was 10 years (range $=0.1-72$ years; IQR=2-20 years). Details of the primary IHR could be obtained only in 21 of the 48 patients before the RIH surgery. Previously diagnosed IH cases included: i) indirect hernia in 5 lesions $(10 \%)$, ii) direct hernia in $16(31 \%)$, iii) femoral hernia in none, iv) combined indirect and direct hernia in one (2\%), and v) unknown in 30 lesions (57\%).

The present system-based classification and its clinical relevance. Intraoperative findings are summarized in Table II. According to the EHSC, we observed: i) indirect RIH in $6(11 \%)$ cases, ii) direct RIH in $42(81 \%)$, iii) femoral hernia in $3(6 \%)$, and iv) combined indirect and direct RIH in 1 $(2 \%)$. The present system-based classification of the recurrence identified: i) $\mathrm{TL}$ in 7 lesions (TL-E $=0$, TL-I $=0$, TE$\mathrm{L}=7$ ), ii) $\mathrm{TD}$ in 5 (TD-E $=0$, TD-I=3, TD-L=2), iii) $\mathrm{MD}$ in 10 (MD-E=3, MD-I=7, MD-L=0), iv) PM in 11 (PM-E=5, PM$\mathrm{I}=6, \mathrm{PM}-\mathrm{L}=0)$, v) $\mathrm{MM}$ in 11 (MM-E=11, MM-I=0, MM-L=0), and vi) $\mathrm{UC}$ in 8 (UC-E=1, UC-I=6, UC-L=1) (Figure 2). The majority of the cases $(81 \%)$ corresponded to the direct RIH 


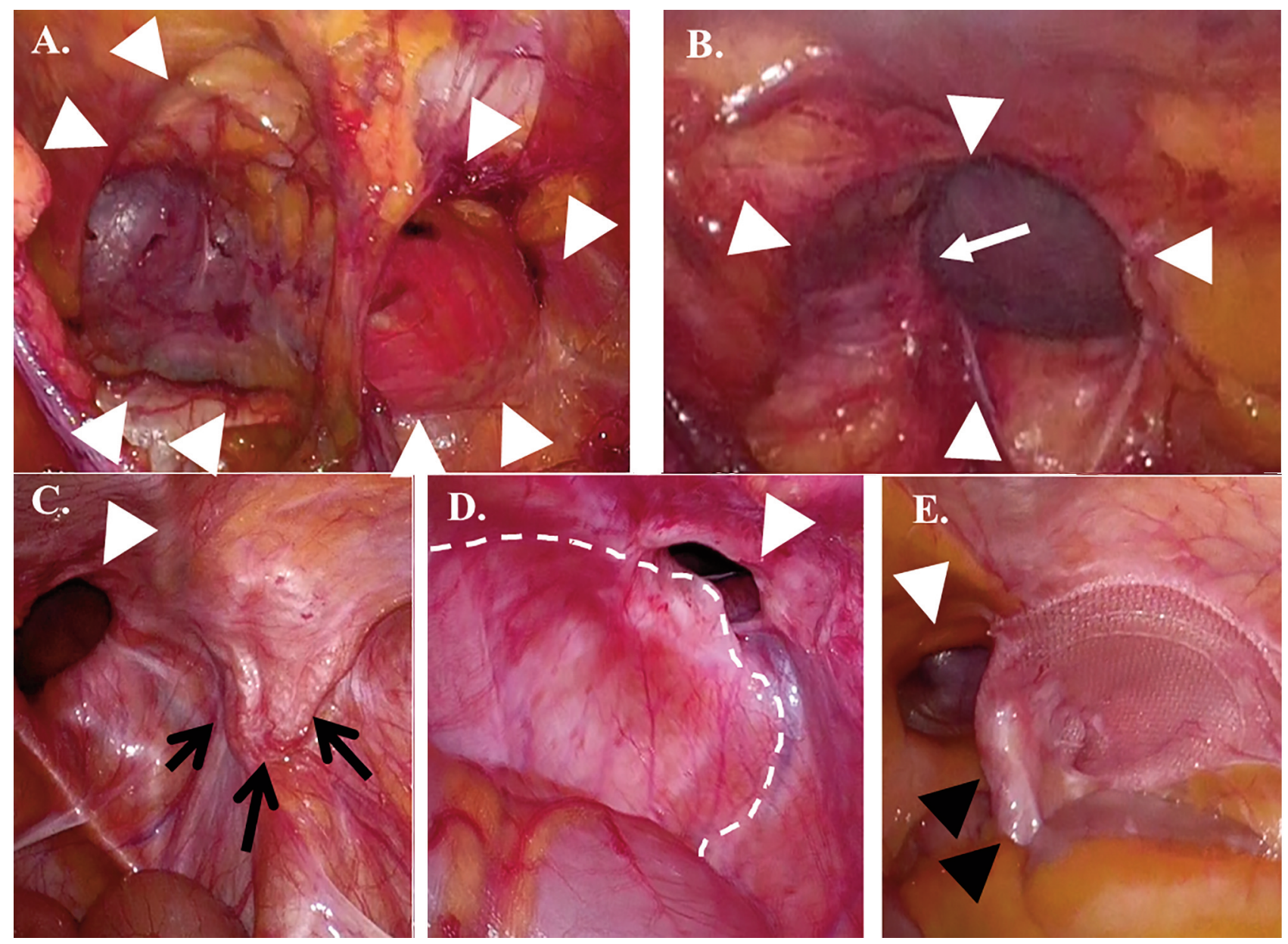

Figure 1. The present classification system. Recurrence after non-mesh suture-repair or the Lichtenstein-patch repair was classified as the tissueloosening type (TL) and the tissue-disruption type (TD) according to whether the continuity of the posterior floor tissue (the transverse fascia and/or internal oblique aponeurosis) was maintained or not. In the TL type, tissue disruption was not observed in the hernia defect (white arrowheads) (Right indirect and direct inguinal hernia, A). In the TD type, the disrupted aponeurosis (white arrow) was observed in the hernia defect (white arrowheads) (Right indirect inguinal hernia, B). Recurrence after the previous open-posterior-mesh-repair was classified according to the locational relation between hernia defect and indwelled mesh as the following: the mesh-distant (MD), the para-mesh (PM) and the mesh migration (MM) types. In the MD type, the hernia defect (white arrowhead) was distant from the indwelled mesh (black arrows) (Right direct inguinal hernia, C). In the PM type, the hernia defect (white arrowhead) was adjacent to the previously indwelled mesh (white dotted line) (Left direct inguinal hernia, D). In the MM type, mesh (black arrowheads) was obviously distorted to be away from the abdominal wall, where the hernia effect is visible (white arrowhead), and migrated from the place where the mesh should have been placed (Right direct inguinal hernia, E).

defined by the EHSC, thus, we could not observe a statistically significant association between the EHSC and the present system-based classification. Moreover, the EHSC does not correlate with the present system time phases.

Cases of a previous LPR were not found. All the 8 UC cases showed a direct $\mathrm{IH}$ and underwent a purely anterior approach surgery without laparoscopy for RIH. Details concerning the previous IHR were unknown in all these 8 cases. Although no tissue disruption was observed, the method of the previous IHR could not be determined from the intraoperative findings alone in these 8 cases, thus, they were classified as the UC type.
The portion of time-phase '-E' was significantly higher in patients undergoing mesh-repair compared to patients undergoing suture-repair during their first IHR surgery (meshrepair $v s$. suture-repair, 20/34 vs. 0/13, $p<0.001$ ). Conversely, the portion of time-phase '- $\mathrm{L}$ ' was significantly greater in patients undergoing suture-repair compared to patients undergoing mesh-repair during their first IHR surgery (suturerepair vs. mesh-repair, 9/13 vs. 0/34, $p<0.001$ ) (Figure 2). MTR was significantly shorter in patients undergoing meshrepair $(n=34)$ compared to patients undergoing suture-repair $(\mathrm{n}=13)$ [mesh-repair $v s$. suture-repair, 1.6 years $(0.1-20) v s$. 30 years (15-72), $p<0.001]$ (Figure 3A). Moreover, MTR 
Table I. Characteristics of the patients.

\begin{tabular}{lc}
\hline Variables & Data \\
\hline Age at surgery for recurrence & 70 years $(32-91)$ \\
Gender (Male/Female) & $47 / 1$ \\
Age at the primary surgery & 60 years $(19-85)$ \\
$\begin{array}{l}\text { Duration from the primary surgery } \\
\text { to recurrence }\end{array}$ & 10 years [0.1-72 (IQR=2-20)] \\
The primary surgery institution & \\
Our hospital/other hospitals & $18 / 34$ \\
EHS classification at the primary surgery & \\
L1-3M0F & $5(9.6 \%)$ \\
L0M1-3F0 & $16(30.8 \%)$ \\
L0M0F1-3 & 0 \\
L1-3M1-3F0 & $1(1.9 \%)$ \\
Unknown & $30(57.7 \%)$ \\
Preoperatively perceived operation methods & \\
for the primary surgery & \\
Tissue-suture repair & $2(3.8 \%)$ \\
Mesh plug & $3(5.8 \%)$ \\
Pre-peritoneal indwelled mesh & $18(34.6 \%)$ \\
Unknown & $28(53.8 \%)$ \\
\hline
\end{tabular}

IQR: Interquartile range; EHS: European Hernia Society; L: lateral/indirect hernia; M: medial/direct hernia; F: femoral hernia.

(years) was significantly shorter in the order of MM $[0.5(0.1-$ 2.0)], PM [2.6 (0.2-15)], MD [11 (0.5-20)], TD [20 (15-30)], and TL [40 (30-72)] $(p<0.001)$ (Figure 3B). Notably, all cases of MM developed within 2 years after the first IHR surgery.

Effect of laparoscopy surgery for recurrent inguinal hernia. The operation method was: i) a completely laparoscopic repair in 19 lesions, ii) the hybrid method in 10 lesions, where the mesh was placed in the preperitoneal space through the anterior approach with the aid of a laparoscope (14), and iii) a purely anterior approach in 23 lesions. Namely, laparoscopy was used in 29 of the 52 lesions (Table II).

The incidence of re-recurrence following RIH surgery was similar between patients undergoing surgery with and without laparoscopy (cumulative re-recurrence rate after the RIH surgery: with $v s$. without laparoscopy: $0 \% v s .5 \%$ in 1 year; $7 \%$ vs. $19 \%$ in 3 years; $7 \%$ vs. $19 \%$ in 5 years, $p=0.270$ ) (Figure 4). Re-recurrence was observed in 4 of the 23 cases that underwent surgery without laparoscopy and in 1 of the 29 cases that underwent laparoscopy surgery. Thus, 5 of the 52 cases developed re-recurrence.

All these five cases underwent a mesh-plug repair without onlay patch at the RIH surgery. Although no obvious hernia defect or intraabdominal content prolapse was observed, the inguinal bulging caused by the prolapse of the mesh-plug itself was apparent. We diagnosed the inguinal bulging as rerecurrence. All these 5 cases underwent LPR for the rerecurrence, with no re-re-recurrence after that observed in any of them.
Table II. Intraoperative variables of the surgery for recurrence.

\begin{tabular}{lc}
\hline Variables & Data \\
\hline EHS Classification & \\
L1-3M0F0 & $6(11.3 \%)$ \\
L0M1-3F0 & $42(80.8 \%)$ \\
L0M0F1-3 & $3(5.8 \%)$ \\
L1-3M1-3F0 & $1(1.9 \%)$ \\
Intraoperatively-proved methods & \\
of the prior surgery & \\
Preperitoneal indwelled mesh & $20(38.5 \%)$ \\
Mesh plug & $15(28.8 \%)$ \\
Tissue-suture repair & $12(23.1 \%)$ \\
Unknowable & $5(9.6 \%)$ \\
The proposed system-based classification & \\
TD (-E/ -I/ -L) & $5(9.6 \%)(0 / 3 / 2)$ \\
TL (-E/ -I/ -L) & $7(13.5 \%)(0 / 0 / 7)$ \\
PM (-E/ -I/ -L) & $11(21.2 \%)(5 / 6 / 0)$ \\
MD (-E/ -I/ -L) & $10(19.2 \%)(3 / 7 / 0)$ \\
MM (-E/ -I/ -L) & $11(21.2 \%)(11 / 0 / 0)$ \\
UC (-E/ -I/ -L) & $8(15.4 \%)(1 / 6 / 1)$ \\
Operation methods for the recurrence & \\
Without laparoscope & \\
Mesh plug & $14(27.1 \%)$ \\
Preperitoneal indwelled mesh & $8(16.7 \%)$ \\
Lichtenstein patch & $1(2.1 \%)$ \\
With laparoscope & \\
Mesh plug & $7(14.5 \%)$ \\
Preperitoneal indwelled mesh & $2(4.2 \%)$ \\
Lichtenstein patch & $1(2.1 \%)$ \\
TAPP & $15(25.0 \%)$ \\
IPOM & $4(6.3 \%)$ \\
\hline
\end{tabular}

EHS: European Hernia Society; L: lateral/indirect hernia; M: medial/direct hernia; F: femoral hernia; TD: tissue disruption type; TL: tissue laxity type; PM: para-mesh type; MD: mesh-distant type; MM: mesh migration type; UT: unclassifiable type; -E: early; -I: intermediate; -L: late; TAPP: transabdominal pre-peritoneal approach repair; IPOM: intra-peritoneal onlay mesh repair.

Regarding the 8 UC cases, all of which underwent a purely anterior approach surgery for the RIH, neither a locational relation between the hernia defect and the mesh was observed during RIH surgery nor a presence of an open-posterior-mesh that might have been used at their first IHR. The portion of the UC was significantly higher in patients who had undergone surgery without laparoscopy than those who had undergone laparoscopy surgery for the RIH [8/23 (34.8\%) vs. $0 / 29(0 \%)$, respectively, $p=0.001]$. Namely, the use of laparoscopy at the RIH surgery did not affect the re-recurrence rate but was significantly advantageous for determining the reliability of the present system-based classification.

\section{Discussion}

In this study, we demonstrated that the MTR was significantly shorter in IH patients that had previously 


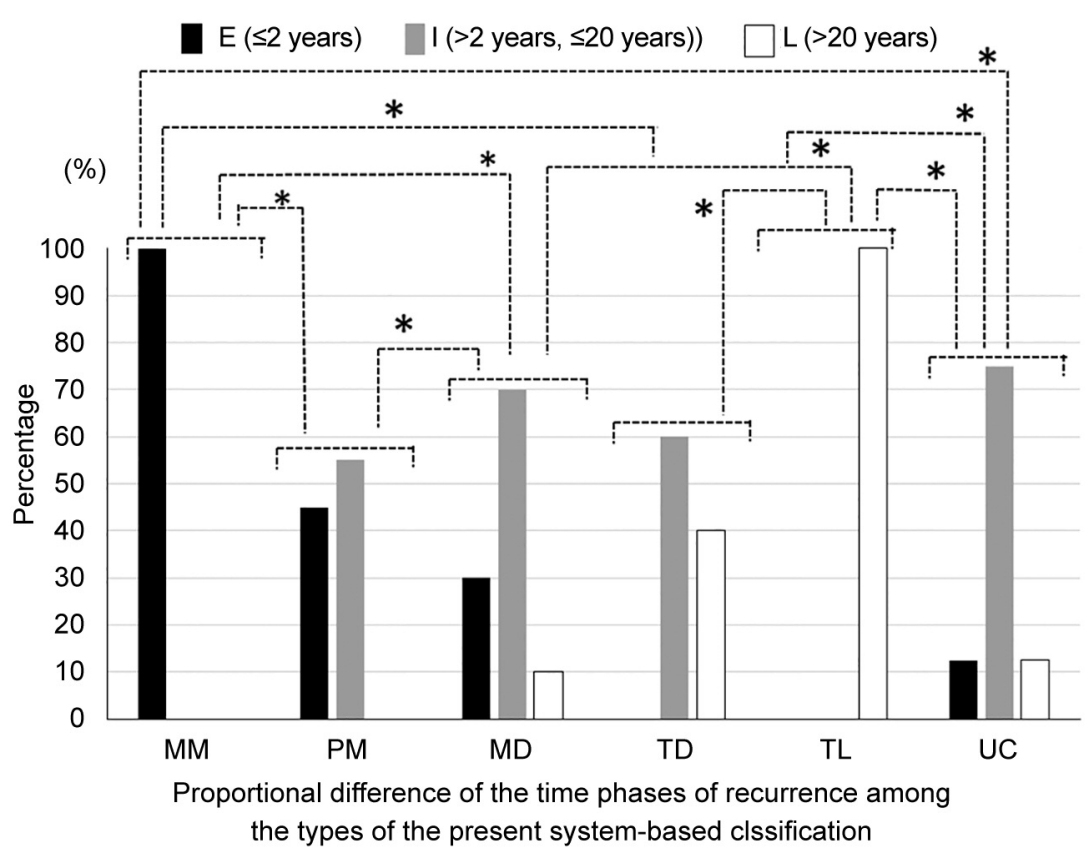

Figure 2. Proportional difference of the time phase of recurrence among the types of the present system-based classification. The proportion of time-phase '-E' was significantly higher in patients having gone through mesh-repair (MM, $P M, M D)$ compared to primary suture-repair (TD, TL) (mesh-repair vs. suture-repair, 20/34 vs. 0/13, p<0.001), and conversely the proportion of the time-phase '- $L$ ' was significantly higher in patients having gone through suture-repair compared to the mesh-repair (Suture-repair vs. mesh-repair, 9/13 vs. 0/34, p<0.001). RIH: Recurrent inguinal hernia; E: early; I: intermediate; L: late; MM: mesh migration; PM: para-mesh; MD: mesh distant; TD: tissue-disruption; TL: tissue-loosening.

undergone mesh-repair than in patients having gone through suture-repair, particularly in the order of MM, PM, MD, TD, and TL. Most notably, all cases of MM developed early, within 2 years, after the first IHR surgery. Based on the above, the following hypotheses were verified by our present findings: i) RIH after mesh-repair develops earlier than the recurrence after suture-repair, ii) RIH caused by the modifiable previous-operation-related factors develops earlier than the recurrence caused by the non-modifiable patient-related factors, and iii) the more inappropriate the mesh handling, the earlier the recurrence.

Currently, the most prevalent classification system of the $\mathrm{IH}$, including the RIH, i.e., the EHSC, does not include factors, such as time-phase of the RIH. In addition, the EHSC does not reflect the methods of the primary surgery at all even when classifying the RIH. Therefore, the EHSC cannot be considered adequate for understanding the mechanisms of developing RIH. The abovementioned factors may only be speculations concerning the development of $\mathrm{RIH}$; however, our present findings support these speculations. We believe that understanding the mechanisms of developing RIH can lead to improved outcomes of future primary open IHR.

A classification system for a condition that most probably necessitates subsequent surgery/ies should be useful for the choice of surgical procedures. From this aspect, the established classification system cannot be used for a definitive choice of treatments, despite the fact that the surgeries for the RIH and the primary IH have little diversity between them $(1,4,6,8-12)$. Both IH and RIH surgeries currently almost always use the mesh, irrespective of whether they are open or laparoscopic. The type of the mesh and the approaching methods (open or laparoscopic) are determined by each surgeon's and/or institution's preference in most occasions (1). Above all, the EHSC cannot dictate the methods of subsequent surgery either for IH or RIH. With all these limitations, we believe that the present classification system suggested here is more useful compared to EHSC for understanding the mechanisms of developing $\mathrm{RIH}$, and can, thus, be used as a quality assessment tool for the primary open IHR.

There are several limitations in this study. First, the timephases of the recurrence were determined simply by a 2 - or 20-year margin, although the RIH has been reported to show a constant linear rise over the years (2, 10-12). Interestingly, most reports showing such a constant linear rise have been reported from institutions, where the method of the primary IHR was nearly always LPR (9-13). In contrast to these reports, there was no case receiving LPR as the primary IHR in this study. Although this may be coincidental, the IQR of 

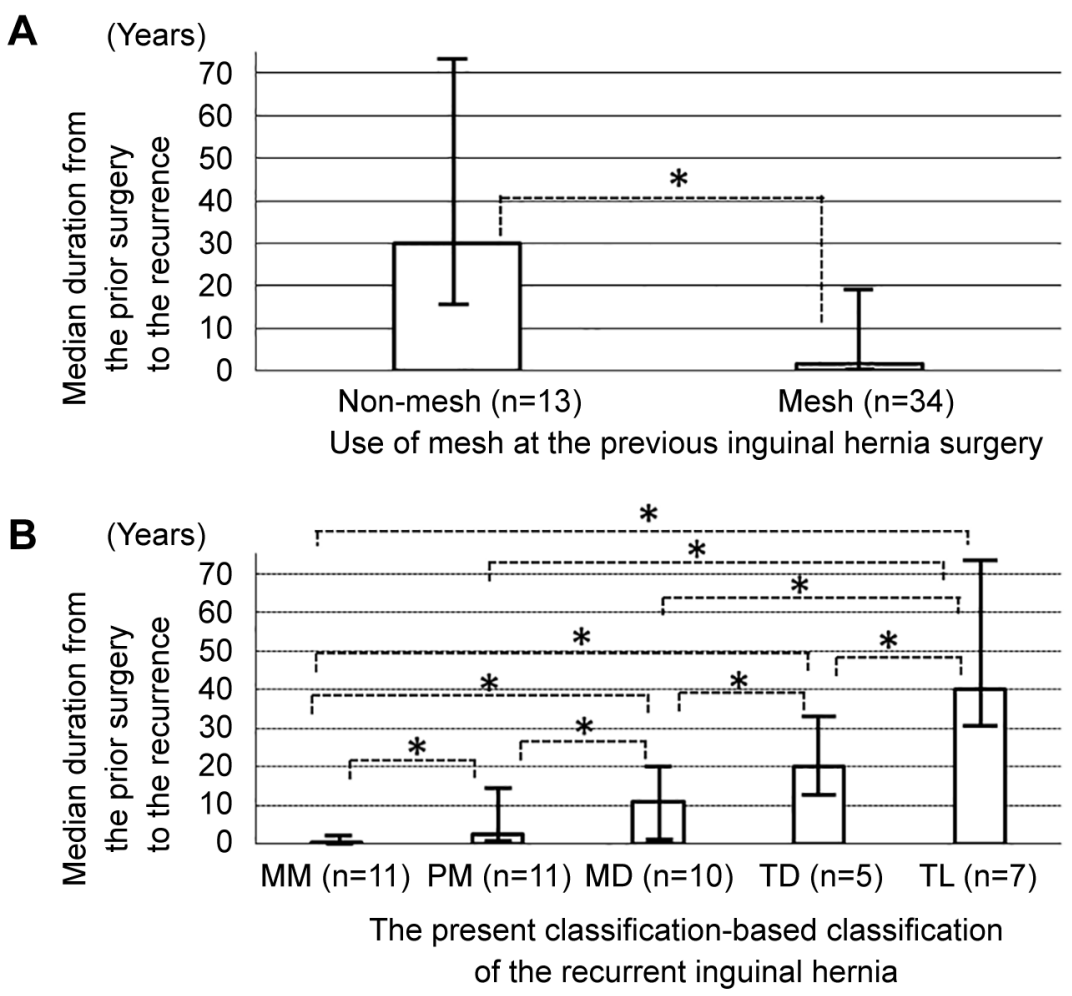

Figure 3. Median time from the primary inguinal hernia repair to the development of the recurrent inguinal hernia. The median time to recurrence (MTR) was significantly shorter in patients undergoing primary mesh-repair $(n=34)$ than in patients undergoing primary suture-repair $(n=13)$ [mesh-repair vs. suture-repair, 1.6 years (0.1-20) vs. 30 years (15-72), $p<0.001]$ (A). MTR (years) was significantly shorter in the order of the mesh migration type (MM) [0.5 (0.1-2.0)], para-mesh type (PM) [2.6 (0.2-15)], mesh-distant type (MD) [11 (0.5-20)], tissue-disruption type (TD) \{[0 $(15-30)]$, and tissue-loosening type (TL) [40 [(30-72)] $(p<0.001)(B)$. Of note, all cases of MM developed within 2 years after the prior surgery.

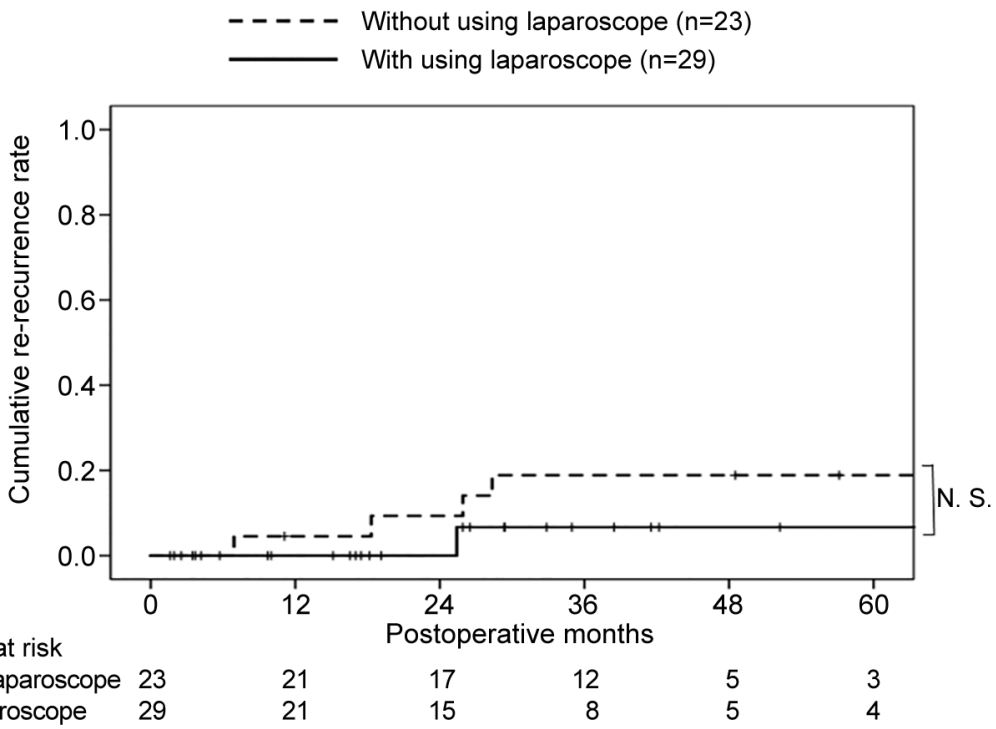

Figure 4. Difference of the cumulative re-recurrence rate between the patients with and without using laparoscope in the surgery for the recurrent inguinal hernia. The incidence of re-recurrence after surgery for it was similar between patients undergoing surgery using laparoscopy or not. The cumulative re-recurrence rate after the surgery for the recurrent inguinal hernia was $0 \%$ at 1-year, 7\% at 3-year, and 7\% at 5-year in patients who underwent surgery using laparoscopy, and 5\% at 1-year, 19\% at 3-year, 19\% at 5-year in patients who underwent surgery without using laparoscopy (p=0.270). 
the time to recurrence in this study ranged between 2 and 20 years. In addition, the fact that significant differences were observed between each time-to-recurrence-related classification group may indicate the validity of the 2- and 20-year range for determining correctly the time-phase for IHR. In addition, the pattern of the time-phases shown in this study, in which some peak-incidence was observed, e.g., all MM type recurrence occurred within 2 years after the first surgery, may demonstrate the characteristics of the recurrence after OPMR. Second, the present classification system appears insufficient for defining the recurrence pattern following LPR. In Japan, the LPR is relatively less prevalent (16). Reflecting this, none of the cases were judged so as to undergo LPR during the primary IHR in this study, even though LPR is the first choice of open IHR in many institutions all over the world (1, 4-6, 11-13, 15, 16). Confirmation of an LPR mesh in place seems easy via the anterior approach, but may be difficult through an exclusively laparoscopic approach. In this study, the presence of an LPR mesh in cases of a completely laparoscopic repair was confirmed by laparoscopic inspection and/or palpation through the hernia defect after exfoliating the parietal peritoneum through a laparoscopic procedure. This confirmation method appears quite uncertain. Thus, the application of the present classification system for cases of a previous LPR requires a more certain method for confirming the existence of the LPR mesh through a completely laparoscopic approach. Third, in contrast to the second limitation, confirming the existence/location of a mesh indwelled in the pre-peritoneal space was quite difficult without the laparoscope. In this study, the number of the UC cases was 8 . All these 8 cases underwent a purely anterior approach surgery. In other words, whether a mesh had been previously used in the primary IHR or not could not be determined through the anterior approach view alone in these 8 cases, and so they were classified as UC. This fact suggests that the laparoscope may be nearly mandatory for applying this classification system to cases where details of the primary IHR are unknown and for determining the locational relation between the mesh and the hernia defect in cases of previous OPMR. Namely, this classification system may be often inapplicable to cases where the use of the laparoscope is undesirable. In fact, this may be the greatest limitation of the present classification system. It has been reported that surgical meshes can be identified quite certainly using magnetic resonance imaging (MRI) (17). In cases where detailed information of the previous IHR is unavailable and the use of the laparoscope is undesirable, MRI may be useful for applying the present classification system.

In conclusion, our findings suggest that our classification system reflects the causes of RIH that are balanced by the non-modifiable and modifiable factors and that the development and time-phases of the RIH are dictated by the interplay between these factors. We believe that this classification system is useful for understanding the mechanisms of developing RIH and could lead to improved outcomes of future primary open IHR.

\section{Conflicts of Interest}

There are no relevant conflicts of interest to report for any authors. No funding sources were used for authors involved with the manuscript.

\section{Authors' Contributions}

NY, DM, MM, and YS had full access to all data in the study and take responsibility for the integrity of data and the accuracy of data analysis. Study concept and design was by NY, YI, DM, KY, NH, NO, RM, YS, and IE. Acquisition of Data was by NY, YI, NO, KY, $\mathrm{NH}, \mathrm{DM}, \mathrm{FA}, \mathrm{RM}$, and YS. Analysis and interpretation of data were by NY, YI, KY, NH, MM, DM, YS, and IE. Drafting of the manuscript was by NY, DM, YI, RM, YS, and IE. Critical revision of the manuscript for important intellectual content was by NY, DM, YI,

\section{Acknowledgements}

We would like to thank all study participants and co-medical staffs for preparing this manuscript. There are no relevant conflicts of interest to report for any authors. No funding sources were used for authors involved with the manuscript. Informed consent was obtained from all individual participants included in this study. This study was approved by the IRB (Approval No. was YEH2014-S$01)$, and was the Declaration of Helsinki compliant.

\section{References}

1 HerniaSurge Group: International guidelines for groin hernia management. Hernia 22(1): 1-165, 2018. PMID: 29330835. DOI: $10.1007 / \mathrm{s} 10029-017-1668-\mathrm{x}$

2 Ashrafi D, Siddaiah-Subramanya M, Memon B and Memon MA: Causes of recurrences after open inguinal herniorrhaphy. Hernia 23(4): 637-645, 2019. PMID: 30519909. DOI: 10.1007/ s10029-018-1868-z

3 Fan CJ, Chien HL, Weiss MJ, He J, Wolfgang CL, Cameron JL, Pawlik TM and Makary MA: Minimally invasive versus open surgery in the Medicare population: a comparison of postoperative and economic outcomes. Surg Endosc 32(9): 3874-3880, 2018. PMID: 29484556. DOI: 10.1007/s00464-018-6126-Z

4 Cavazzola LT and Rosen MJ: Laparoscopic versus open inguinal hernia repair. Surg Clin North Am 93(5): 1269-1279, 2013. PMID: 24035088. DOI: 10.1016/j.suc.2013.06.013

5 Reinpold W: Risk factors of chronic pain after inguinal hernia repair: a systematic review. Innov Surg Sci 2(2): 61-68, 2017. PMID: 31579738. DOI: 10.1515/iss-2017-0017

6 Wu JJ, Way JA, Eslick GD and Cox MR: Transabdominal preperitoneal versus open repair for primary unilateral inguinal hernia: a meta-analysis. World J Surg 42(5): 1304-1311, 2018. PMID: 29075859. DOI: 10.1007/s00268-017-4288-9

7 Miller HJ: Inguinal hernia: mastering the anatomy. Surg Clin North Am 98(3): 607-621, 2018. PMID: 29754625. DOI: 10.1016/j.suc.2018.02.005 
8 Primatesta $\mathrm{P}$ and Goldacre MJ: Inguinal hernia repair: incidence of elective and emergency surgery, readmission and mortality. Int J Epidemiol 25(4): 835-839, 1996. PMID: 8921464. DOI: 10.1093/ije/25.4.835

9 Keller JJ, Muo CH, Lan YC, Sung FC, Lo FE, Chiang KY, Lyu SY and Tsai ST: A nation-wide population-based study of inguinal hernia repair incidence and age-stratified recurrence in an Asian population. Hernia 19(5): 735-740, 2015. PMID: 25739714. DOI: 10.1007/s10029-015-1359-4

10 Lederhuber H, Hanßke B and Dahlstrand U: Impact of trainee participation on inguinal hernia repair outcome: a study based on the Swedish hernia register. Ann Surg 274(1): e62-e69, 2021. PMID: 31365364. DOI: 10.1097/SLA.0000000000003497

11 Burcharth J, Andresen K, Pommergaard HC, Bisgaard T and Rosenberg J: Recurrence patterns of direct and indirect inguinal hernias in a nationwide population in Denmark. Surgery 155(1): 173-177, 2014. PMID: 24694361. DOI: 10.1016/j.surg. 2013.06.006

12 Murphy BL, Ubl DS, Zhang J, Habermann EB, Farley DR and Paley K: Trends of inguinal hernia repairs performed for recurrence in the United States. Surgery 163(2): 343-350, 2018. PMID: 28923698. DOI: 10.1016/j.surg.2017.08.001

13 Burcharth J: The epidemiology and risk factors for recurrence after inguinal hernia surgery. Dan Med J 61(5): B4846, 2014. PMID: 24814748.
14 Tsukada T, Saito H, Amaya K, Terada I, Kaji M and Shimizu K: Hybrid laparoscopic and anterior approach for postsurgical inguinal hernia after iliofemoral arterial bypass. Int Surg 100(3): 431-435, 2015. PMID: 25785323. DOI: 10.9738/INTSURG-D14-00226.1

15 Pisanu A, Podda M, Saba A, Porceddu G and Uccheddu A: Meta-analysis and review of prospective randomized trials comparing laparoscopic and Lichtenstein techniques in recurrent inguinal hernia repair. Hernia 19(3): 355-366, 2015. PMID: 25033943. DOI: $10.1007 / \mathrm{s} 10029-014-1281-1$

16 Takata H, Matsutani T, Hagiwara N, Ueda J, Arai H, Yokoyama $\mathrm{Y}$, Nomura $\mathrm{T}$ and Uchida $\mathrm{E}$ : Assessment of the incidence of chronic pain and discomfort after primary inguinal hernia repair. J Surg Res 206(2): 391-397, 2016. PMID: 27884334. DOI: $10.1016 /$ j.jss.2016.08.027

17 Hansen NL, Ciritsis A, Otto J, Busch D, Kuhl CK and Kraemer NA: Utility of magnetic resonance imaging to monitor surgical meshes: Correlating imaging and clinical outcome of patients undergoing inguinal hernia repair. Invest Radiol 50(7): 436-442, 2015. PMID: 25756683. DOI: 10.1097/RLI.0000000000000148

Received January 3, 2021

Revised August 24, 2021

Accepted August 27, 2021 